\title{
Multi-linear Secret-Sharing Schemes
}

\author{
Amos Beimel $^{1, \star}$, Aner Ben-Efraim ${ }^{1,2, \star}$, Carles Padró $^{3}$, and Ilya Tyomkin ${ }^{2, \star \star}$ \\ 1 Dept. of Computer Science, Ben Gurion University of the Negev, Be'er Sheva, Israel \\ 2 Dept. of Mathematics, Ben Gurion University of the Negev, Be'er Sheva, Israel \\ ${ }^{3}$ Nanyang Technological University, Singapore
}

\begin{abstract}
Multi-linear secret-sharing schemes are the most common secret-sharing schemes. In these schemes the secret is composed of some field elements and the sharing is done by applying some fixed linear mapping on the field elements of the secret and some randomly chosen field elements. If the secret contains one field element, then the scheme is called linear. The importance of multi-linear schemes is that they provide a simple non-interactive mechanism for computing shares of linear combinations of previously shared secrets. Thus, they can be easily used in cryptographic protocols.

In this work we study the power of multi-linear secret-sharing schemes. On one hand, we prove that ideal multi-linear secret-sharing schemes in which the secret is composed of $p$ field elements are more powerful than schemes in which the secret is composed of less than $p$ field elements (for every prime $p$ ). On the other hand, we prove super-polynomial lower bounds on the share size in multi-linear secret-sharing schemes. Previously, such lower bounds were known only for linear schemes.
\end{abstract}

Keywords: Ideal secret-sharing schemes, multi-linear matroids, Dowling geometries.

\section{Introduction}

Consider a scenario where a user holds some secret information and wants to store it on some servers such that only some predefined sets of servers (i.e., trusted sets) can reconstruct this information. Secret-sharing schemes enable such storage, where the dealer - the user holding the secret - computes some strings, called shares, and privately gives one share to each server. In the sequence we will refer to the servers as the parties and to the collection of sets of parties that can reconstruct the secret as an access structure. Secret-sharing schemes are an important cryptographic primitive and they are used nowadays as a basic

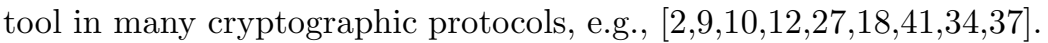

In this work we study the most useful construction of secret-sharing schemes, namely, multi-linear secret-sharing schemes. In these schemes the secret is a

\footnotetext{
* Partially supported by ISF grants 938/09, 544/13 and by the Frankel Center for Computer Science.

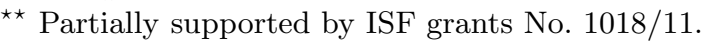

Y. Lindell (Ed.): TCC 2014, LNCS 8349, pp. 394418 2014.

(C) International Association for Cryptologic Research 2014
} 
sequence of elements from some finite field, and each share is a linear combination of these elements and some random elements from the field. If the secret contains exactly one element of the field, then the scheme is called linear. Linear and multi-linear secret-sharing schemes are very useful as they provide a simple noninteractive mechanism for computing shares of linear combinations of previously shared secrets.

We prove two results on the power of multi-linear secret-sharing schemes. Our first results shows advantages of multi-linear secret-sharing schemes compared to linear schemes, that is, we prove that ideal schemes in which the secret contains $p$ elements of the field are more efficient than schemes in which the secret contains less than $p$ field elements (for every prime $p$ ). Our second results proves super polynomial lower bounds on the size of shares in multi-linear secret-sharing schemes.

Previous Results. Threshold secret-sharing schemes, where all sets of parties whose size is at least some threshold, were introduced by Shamir 33. and Blakley [5]. Secret-sharing schemes for general access structures were introduced and constructed by Ito et al. [19. Better constructions were introduced by Benaloh and Leichter [3]. Linear secret-sharing schemes were presented by Brickel [7] for the case that each share is one field element and by Krachmer and Wigderson 20] for the case that each share can contain more than one field element. Karchmer and Wigderson's motivation was studying a complexity model called span programs; in particular, they proved that monotone span programs are equivalent to linear secret-sharing schemes. It is important to note that all previously mentioned constructions of secret-sharing schemes are linear. Multi-linear secret-sharing schemes were studied by [413, who gave the conditions when a multi-linear scheme realizes an access structure. Construction of multi-linear secret-sharing schemes were given by, e.g., [36 6 39 38].

To explain why linear secret-sharing schemes are useful, we describe the basic idea in using secret-sharing schemes in protocols, starting from [2]. In such protocols the parties share their inputs among the other parties, and, thereafter, the shares of different secrets are "combined" to produce shares of some function of the original secrets. For example, the parties hold shares of two secrets $a$ and $b$, and they want to compute shares of $a+b$ (without reconstructing the original secrets). If the schemes are multi-linear, the two secrets $a$ and $b$ are shared using the same multi-linear scheme, and each party sums the shares of the two secrets, then the resulting shares are of the secret $a+b$.

In any secret-sharing scheme, the size of the share of each party is at least the size of the secret [21]. An ideal secret-sharing scheme is a scheme in which the size of the share of each party is exactly the size of the secret. For example, Shamir's scheme [33] is ideal. Brickell [7] considered ideal schemes and constructed ideal schemes for some access structures, e.g., for hierarchical access structures. Brickell and Davenport [8] showed an interesting connection between ideal access structures and matroids, that is, (1) If an access structure is ideal then it is induced by a matroid, (2) If an access structure is induced by a representable matroid, then the access structure is ideal. Following this work, 
many works have studied ideal access structures and matroids, e.g. $32|35| 25 \mid 24$. In particular, if an access structure is induced by a multi-linear representable matroid, then it is ideal 35 .

Simonis and Ashikhmin [35] considered the access structure induced by the Non-Pappus matroid. They construct an ideal multi-linear secret-sharing scheme realizing this access structure, where the secret contains two field elements, and they prove (using known results about matroids) that there is no ideal linear secret-sharing realizing this access structure (that is, in any linear secret-sharing realizing this access structure at least one share must contain more than one field element). Pendavingh and van Zwam [29] (implicitly) provided another example of an access structure that can be realized by an ideal multi-linear secret-sharing scheme, where the secret contains two field elements, but cannot be realized by an ideal linear secret-sharing scheme. Their example is the access structure induced by the rank-3 Dowling matroid of the quaternion group. Note that the rank-3 Dowling matroid 1514 can be defined with an arbitrary group (see Definition 2.9); in this paper we will use it with properly chosen groups.

For a scheme to be efficient and useful, the size of the shares should be small (i.e., polynomial in the number of parties). The best known schemes for general access structures, e.g., [1932013], are highly inefficient, that is, for most access structures the size of shares is $2^{O(n)}$ times the size of the secret, where $n$ is the number of parties in the access structure. The best lower bound known on the total share size for an access structure is $\Omega\left(n^{2} / \log n\right)$ times the size of the secret [1]. Thus, there exists a large gap between the known upper and lower bounds. Bridging this gap is one of the most important questions in the study of secret-sharing schemes. In contrast to general secret-sharing schemes, super-polynomial lower bounds are known for linear secret-sharing schemes. That is, there exist explicit access structures such that the total share size of any linear secret-sharing scheme realizing them is $n^{\Omega(\log n)}$ times the size of the secret 11617 .

Our Results and Techniques. The simplest way to construct a multi-linear secretsharing scheme, where the secret is composed of $k$ field elements, is to share each field element independently using a linear secret-sharing scheme. This results in a multi-linear scheme whose information ratio (the ratio between the length of the shares and the length of the secret) is the same as the information ratio of the linear scheme. The question is if one can construct multi-linear secretsharing schemes whose information ratio is better than linear schemes. Our first result gives a positive answer to this question. Our second result implies that in certain cases the answer is no - we show that the lower bound of [17] for linear secret-sharing schemes holds also for multi-linear secret-sharing schemes.

Our first results shows advantages of multi-linear secret-sharing schemes compared to linear schemes. For every prime $p>2$, we show that there is an access structure such that: (1) It has an ideal multi-linear secret-sharing scheme in which the secret is composed of $p$ field elements. (2) It does not have an ideal multi-linear secret-sharing scheme in which the secret is composed of $k$ field elements, for every $k<p$. In other words, we prove that schemes in which the 
secret is composed of $p$ field elements are more efficient than schemes in which the secret is composed of less than $p$ field elements. Previously, this was known only for $p=2$.

To prove this result we consider the access structures induced by rank-3 Dowling matroids of various groups. By known results, it suffices to study when these matroids are $k$-linearly representable. We study this question and show that it can be answered using tools from representation theory. The important step in our proof is showing that the Dowling matroid of a group $G$ is $k$-linearly representable if and only if the group $G$ has a fixed-point free representation of dimension $k$ (see Section 2.5 for definition of these terms). To complete our proof, we show that for every $p$ there is a group $G_{p}$ that has a fixed-point free representation of dimension $p$ and does not have a fixed-point free representation of dimension $k<p$.

Our second results is super polynomial lower bounds on the size of shares in multi-linear secret-sharing schemes. Prior to our work, such lower bounds were known only for linear secret-sharing schemes. As proving super polynomial lower bounds for general secret-sharing schemes is a major open question, any extension of the lower bounds to a broader class of schemes is important. Specifically, as the class of multi-linear secret-sharing schemes is the class that is useful for applications, it is interesting to prove lower bounds for this class. We show that the method of Gál and Pudlák [17] for proving lower bounds for linear secretsharing schemes applies also to multi-linear secret-sharing schemes. As a result, we get that there exist access structures such that the total share size of any multi-linear secret-sharing scheme realizing them is $n^{\Omega(\log n)}$ times the size of the secret (even when the secret contains any number of field elements).

\section{Preliminaries}

Notations. We will frequently use block matrices throughout this paper. To differentiate these block matrices, they will be inside square brackets, or in bold letters (e.g. $\mathbf{A}=\left[\begin{array}{ll}A & B \\ C & D\end{array}\right]$, where $A, B, C, D$ are matrices). In all the proofs and examples, except in the proof of Theorem 4.5, all blocks are of size $k \times k$. For a matrix $A$, we denote the $i^{\text {th }}$ column of $A$ by $A_{i}$. We denote fields by $\mathbb{F}$ or $\mathbb{E}$ (general fields), $\mathbb{C}$ (complex numbers), $\widetilde{\mathbb{F}}$ (algebraic closure of $\mathbb{F}$ ), and $\mathbb{F}_{p^{m}}$ (the unique field with $p^{m}$ elements). We denote the integers by $\mathbb{Z}$ and the non-negative integers by $\mathbb{N}$.

\subsection{Secret-Sharing Schemes}

A secret-sharing scheme is, informally, an algorithm in which a dealer distributes a secret to a set of parties in such that only authorized subsets of parties can reconstruct the secret, while unauthorized subsets cannot learn anything about the secret. We next define secret-sharing schemes, starting with some notations. 
Definition 2.1. Let $\left\{p_{1}, \ldots, p_{n}\right\}$ be a set of parties. $A$ collection $\mathcal{A} \subseteq 2^{\left\{p_{1}, \ldots, p_{n}\right\}}$ is monotone if $B \in \mathcal{A}$ and $B \subseteq C$ imply that $C \in \mathcal{A}$. An access structure is a monotone collection $\mathcal{A} \subseteq 2^{\left\{p_{1}, \ldots, p_{n}\right\}}$ of non-empty subsets of $\left\{p_{1}, \ldots, p_{n}\right\}$. Sets in $\mathcal{A}$ are called authorized, and sets not in $\mathcal{A}$ are called unauthorized.

Definition 2.2 (secret-sharing). A secret-sharing scheme $\Sigma$ with domain of secrets $S$ is a pair $\Sigma=\langle\Pi, \mu\rangle$, where $\mu$ is a probability distribution on some finite set $R$ called the set of random strings and $\Pi$ is a mapping from $S \times R$ to a set of $n$-tuples $K_{1} \times K_{2} \times \cdots \times K_{n}$, where $K_{j}$ is called the domain of shares of $p_{j}$. A dealer distributes a secret $s \in S$ according to $\Sigma$ by first sampling a random string $r \in R$ according to $\mu$, and applying the mapping $\Pi$ on $s$ and $r$, that is, computing a vector of shares $\Pi(s, r)=\left(s_{1}, \ldots, s_{n}\right)$, and privately communicating each share $s_{j}$ to party $p_{j}$. For a set $A \subseteq\left\{p_{1}, \ldots, p_{n}\right\}$, we denote $\Pi_{A}(s, r)$ as the restriction of $\Pi(s, r)$ to its $A$-entries.

Correctness. The secret $s$ can be reconstructed by any authorized set of parties. That is, for any set $B \in \mathcal{A}$ (where $B=\left\{p_{i_{1}}, \ldots, p_{i_{|B|}}\right\}$ ), there exists a reconstruction function $\operatorname{RECON}_{B}: K_{i_{1}} \times \ldots \times K_{i_{|B|}} \rightarrow S$ such that for every $s \in S$,

$$
\operatorname{Pr}\left[\operatorname{RECON}_{B}\left(\Pi_{B}(s, r)\right)=s\right]=1 .
$$

Perfect Privacy. Every unauthorized set cannot learn anything about the secret (in the information theoretic sense) from their shares. Formally, for any set $T \notin \mathcal{A}$, for every two secrets $a, b \in S$, and for every possible vector of shares $\left\langle s_{j}\right\rangle_{p_{j} \in T}$ :

$$
\operatorname{Pr}\left[\Pi_{T}(a, r)=\left\langle s_{j}\right\rangle_{p_{j} \in T}\right]=\operatorname{Pr}\left[\Pi_{T}(b, r)=\left\langle s_{j}\right\rangle_{p_{j} \in T}\right] .
$$

The information ratio of a secret-sharing scheme is $\frac{\max _{1 \leq j \leq n} \log \left|K_{j}\right|}{\log |S|}$, where $S$ is the domain of secrets and $K_{j}$ is the domain of shares of $p_{j}$.

In every secret-sharing scheme, the information ratio is at least 1 [21. Ideal secret-sharing schemes are those where the information ratio is exactly 1 , which means that the size of the domain of the shares is exactly the size of the domain of the secret.

Multi-linear secret-sharing schemes are schemes in which the computation of the shares is a linear mapping. More formally, in a multi-linear secret-sharing scheme over a finite field $\mathbb{F}$, the secret is a vector of elements of the field. To share a secret $s \in \mathbb{F}^{k}$, the dealer first chooses a random vector $r \in \mathbb{F}^{m}$ with uniform distribution (for some integer $m$ ). Each share is a vector over the field such that each coordinate of this vector is some fixed linear combination of the coordinates of the secret $s$ and the coordinates of the random string $r$.

\subsection{Matroids}

Matroids are combinatorial objects that can be defined in many equivalent ways. To make things simple, we will stick to one definition based on rank function. 
Definition 2.3. A matroid $M$ is an ordered pair $(E, r)$ with $E$ a finite set (usually $E=\{1, \ldots, n\}$ ) called the ground set and a rank function $r: 2^{E} \rightarrow \mathbb{N}$ satisfying the following conditions, called the matroid axioms:

1. $r(\emptyset)=0$,

2. If $X \subseteq E$ and $x \in E$, then $r(X) \leq r(X \cup\{x\}) \leq r(X)+1$,

3. If $X \subseteq E$ and $x, y \in E$ such that $r(X \cup\{x\})=r(X \cup\{y\})=r(X)$ then $r(X \cup\{x\} \cup\{y\})=r(X)$.

$A$ set $X \subseteq E$ is independent if $r(X)=|X|$, otherwise $X$ is dependent. The rank of the matroid is defined $r(M):=r(E)$. A base of $M$ is an independent set $X \subseteq E$ such that $r(X)=r(M)$. The set of bases of a matroid uniquely identifies the matroid. A circuit is a minimal dependent set. The set of all circuits of a matroid also uniquely identifies the matroid. Throughout this paper we will assume that every set $X \subseteq E$ of size 2 is independent (called simple matroids or geometries in the literature).

The simplest example of a matroid is the size of a group, i.e., let $E=\{1, \ldots, n\}$ and $r(X)=|X|$. The 3 axioms are trivially verified. In this matroid, all sets are independent. Matroids originated from trying to generalize axioms in graph theory and linear algebra.

Example 2.4. Let $E=\left\{v_{1}, \ldots, v_{n}\right\}$ be a set of vectors over some field $\mathbb{F}$. For $X \subseteq E$ let $r(X)=\operatorname{dim}(\operatorname{span}(X))$. By linear algebra, the 3 matroid axioms hold. Furthermore, we can look at the matrix $A$, in which the $i^{\text {th }}$ column is the vector $v_{i}$. In this case, $r(X)$ is the rank of the submatrix containing the columns of the vectors in $X$. Matroids that arise in this manner are called linearly representable (over $\mathbb{F}$ ). This can also be generalized as follows:

Definition 2.5. Let $M=(E=\{1, \ldots, n\}, r)$ be a matroid and $\mathbb{F}$ a field. $A k$ linear representation of $M$ over $\mathbb{F}$ is a matrix $A$ with $k \cdot n$ columns $A_{1}, \ldots, A_{k \cdot n}$ such that the rank of every set $X=\left\{i_{1}, \ldots, i_{j}\right\} \subseteq E$ satisfies

$$
r(X)=\frac{\operatorname{dim}\left(\operatorname{span}\left(U_{i_{1}} \cup \cdots \cup U_{i_{j}}\right)\right)}{k},
$$

where $U_{\ell}=\left\{A_{(\ell-1) \cdot k+1}, A_{(\ell-1) \cdot k+2}, \ldots, A_{\ell \cdot k}\right\}$ for $1 \leq \ell \leq n$. If such a representation of $M$ exists then $M$ is $k$-linearly representable. One-linearly represetable matroids are called linearly representable. A matroid is multi-linearly representable if it is $k$-linearly representable for some $k \in \mathbb{N}$.

An example of a multi-linear representation is given in Example 2.6.

Matroids of rank 3 can be expressed by a geometric representation on a plane as follows - the bases are the sets of 3 points that are not on a single line. For a diagram on the plane to represent a matroid it must satisfy the following condition: Every 2 distinct points lie on a single line. Since every 2 points lie on a line, usually only lines that pass through at least 3 points are drawn. See [28, Chapter 1.5] for more details and the more general statement. 
Example 2.6. Let $A$ and $\mathbf{B}$ be the following matrix and block matrix:

$$
\begin{aligned}
& \begin{array}{llllllllllll}
1 & 2 & 3 & g_{1}^{\prime} & g_{1}^{\prime \prime} & g_{1}^{\prime \prime \prime} & 1 & 2 & 3 & g_{1}^{\prime} & g_{1}^{\prime \prime} & g_{1}^{\prime \prime \prime}
\end{array} \\
& A=\left(\begin{array}{cccccc}
1 & 0 & 0 & -1 & 0 & 1 \\
0 & 1 & 0 & 1 & -1 & 0 \\
0 & 0 & 1 & 0 & 1 & -1
\end{array}\right), \mathbf{B}=\left[\begin{array}{cccccc}
I_{k} & 0 & 0 & -I_{k} & 0 & I_{k} \\
0 & I_{k} & 0 & I_{k} & -I_{k} & 0 \\
0 & 0 & I_{k} & 0 & I_{k} & -I_{k}
\end{array}\right] \text {. }
\end{aligned}
$$

For any field $\mathbb{F}$, the matrix $A$ (resp. the block matrix $\mathbf{B}$ ) is a linear ( $k$-linear) representation of the matroid with 6 points whose geometric representation is Figure 1 (a). For example, the columns labelled by $1,2, g_{1}^{\prime \prime}$ are independent. Therefore, they do not lie on the same line in Figure 1 (a). On the other hand, the columns labelled by $1,2, g_{1}^{\prime}$ are dependent, thus, they lie on a line.

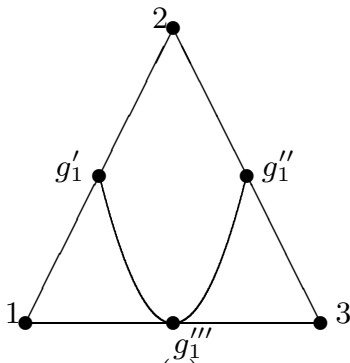

$(a)$

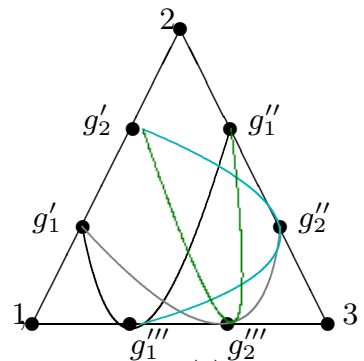

(b)

Fig. 1. Geometric Representation of the matroids $Q_{3}(\{1\})$ and $Q_{3}\left(\mathbb{Z}_{2}\right)$

Definition 2.7. Let $M$ be a matroid and $\mathbb{F}$ a field. We say that that $M$ is $k$ minimally representable over $\mathbb{F}$ if there is a $k$-linear representation of $M$ over $\mathbb{F}$, but for every $j<k$ there is no $j$-linear representation of $M$ over $\mathbb{F}$. We will say that $M$ is $k$-minimally representable if it is $k$-minimally representable over some field $\mathbb{F}$, but not $j$-linearly representable over any field for $j<k$.

Example 2.8. The Non-Pappus matroid (cf. [28, Example 1.5.15, page 39]) whose geometric representation appears in Figure 2 is not linearly representable over any field [28, Proposition 6.1.10], but has a 2-linear representation over $\mathbb{F}_{3}[35$. Therefore, the Non-Pappus matroid is 2-minimally representable.

Our primary focus in the first part of the paper will be the multi-linear representability of the rank-3 Dowling Matroids. These matroids were presented by Dowling [1514. We will show that for every prime $p$ there is a Dowling Matroid which is $p$-minimally representable, and furthermore, over a relatively small field. The Dowling Matroid is defined as follows:

Definition 2.9. Let $G=\left\{1_{G}=g_{1}, g_{2}, \ldots, g_{n}\right\}$ be a finite group. The rank3 Dowling Matroid of $G$, denoted $Q_{3}(G)$, is a matroid of rank 3 on the set $E=\left\{1,2,3, g_{1}^{\prime}, \ldots, g_{n}^{\prime}, g_{1}^{\prime \prime}, \ldots, g_{n}^{\prime \prime}, g_{1}^{\prime \prime \prime}, \ldots, g_{n}^{\prime \prime \prime}\right\}$. That $i s$, for every element 


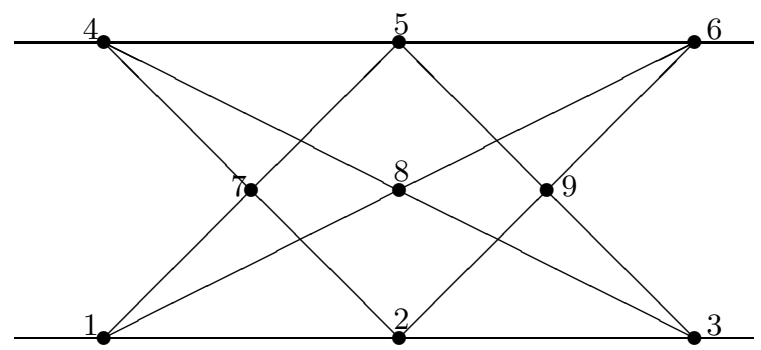

Fig. 2. The Non-Pappus matroid

$g_{i} \in G$, there are 3 elements in the ground set of the matroid $g_{i}^{\prime}, g_{i}^{\prime \prime}, g_{i}^{\prime \prime \prime} \in E$ and there are 3 additional ground set elements 1,2,3 not related to the group. Every subset of 3 elements not in $C_{1} \cup C_{2} \cup C_{3} \cup C_{4}$ is a base of the matroid, where,

$C_{1}=\left\{\left\{1,2, g_{i}^{\prime}\right\} \mid 1 \leq i \leq n\right\} \cup\left\{\left\{1, g_{i}^{\prime}, g_{j}^{\prime}\right\} \mid 1 \leq i<j \leq n\right\} \cup\left\{\left\{2, g_{i}^{\prime}, g_{j}^{\prime}\right\} \mid 1 \leq i<j \leq n\right\}$,

$C_{2}=\left\{\left\{2,3, g_{i}^{\prime \prime}\right\} \mid 1 \leq i \leq n\right\} \cup\left\{\left\{2, g_{i}^{\prime \prime}, g_{j}^{\prime \prime}\right\} \mid 1 \leq i<j \leq n\right\} \cup\left\{\left\{3, g_{i}^{\prime \prime}, g_{j}^{\prime \prime}\right\} \mid 1 \leq i<j \leq n\right\}$,

$C_{3}=\left\{\left\{1,3, g_{i}^{\prime}\right\} \mid 1 \leq i \leq n\right\} \cup\left\{\left\{1, g_{i}^{\prime \prime \prime}, g_{j}^{\prime \prime \prime}\right\} \mid 1 \leq i<j \leq n\right\} \cup\left\{\left\{3, g_{i}^{\prime \prime \prime}, g_{j}^{\prime \prime \prime}\right\} \mid 1 \leq i<j \leq n\right\}$, $C_{4}=\left\{\left\{g_{i}^{\prime}, g_{j}^{\prime \prime}, g_{\ell}^{\prime \prime \prime}\right\} \mid g_{j} \cdot g_{i} \cdot g_{\ell}=1\right\}$.

Alternatively, it can be defined by the geometric representation appearing in Figure [3, with additional lines that go through points $g_{i}^{\prime}, g_{j}^{\prime \prime}, g_{\ell}^{\prime \prime \prime}$ if and only if $g_{j} \cdot g_{i} \cdot g_{\ell}=1_{G}$ (e.g., there is always a line that goes through $g_{1}^{\prime}, g_{1}^{\prime \prime}, g_{1}^{\prime \prime \prime}$ since $g_{1}=1_{G}$ and $\left.1_{G} \cdot 1_{G} \cdot 1_{G}=1_{G}\right)$ 1

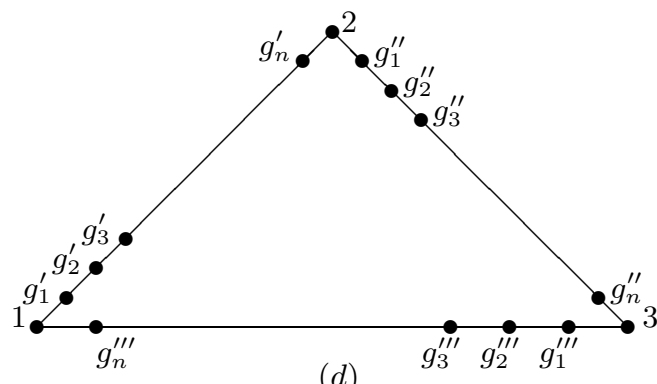

Fig. 3. The Rank-3 Dowling matroid with the lines corresponding to sets $\left\{g_{i}^{\prime}, g_{j}^{\prime \prime}, g_{\ell}^{\prime \prime \prime}\right\}$ such that $g_{j} \cdot g_{i} \cdot g_{\ell}=1_{G}$ missing

We note that the matroid in Example 2.6 is the Dowling matroid of the trivial group. Figure 1 (b) is a geometric representation of the Dowling matroid of the group $\mathbb{Z}_{2}$, the unique group with 2 elements. Dowling [15]14] showed that $Q_{3}(G)$

${ }^{1}$ In the literature, the matroid is sometimes defined a bit differently, e.g., a line goes through $g_{i}^{\prime}, g_{j}^{\prime \prime}, g_{\ell}^{\prime \prime \prime}$ if and only if $\left(g_{j}\right)^{-1} \cdot\left(g_{i}\right)^{-1} \cdot g_{\ell}=1_{G}$. This is just a different naming of the ground set elements. 
is linearly representable over $\mathbb{F}$ if and only if $G$ is isomorphic to a subgroup of $\mathbb{F}^{*}$, the group of invertible elements in $\mathbb{F}$. Our main theorem generalizes this statement for multi-linear representability. Other forms of representability of $Q_{3}(G)$, namely representability over partial fields and skew partial fields, have been studied by Semple and Whittle [30] and Pendavingh and Van Zwam [29].

\subsection{Ideal Secret-Sharing Schemes and Matroids}

There is a strong connection between secret-sharing schemes and matroids. Every matroid with ground set $E=\left\{p_{0}, p_{1}, \ldots, p_{n}\right\}$ induces an access structure $\mathcal{A}$ with $n$ parties $E^{\prime}=\left\{p_{1}, \ldots, p_{n}\right\}$ by the rule $\forall A \subseteq E^{\prime}, A \in \mathcal{A}$ if and only if $r\left(A \cup\left\{p_{0}\right\}\right)=r(A)$. The access structure $\mathcal{A}$ is also known as the matroid port. In a sense, we think of $p_{0}$ as the dealer. Brickell and Davenport [8] showed that all access structures admitting ideal secret-sharing schemes are induced by matroids. However, not all access structures induced by matroids are ideal [32] 25. The class of matroids inducing ideal access structures are called secret-sharing matroids and also almost affinely representable, and discussed in [35. Every multilinearly representable matroid is a secret-sharing matroid. It is still open whether this inclusion is proper. There is also a strong connection between ideal multilinear secret-sharing schemes and multi-linearly representable matroids [20]13].

Proposition 2.10. The class of access structures induced by multi-linearly representable matroids is exactly the access structures admitting an ideal multilinear secret-sharing scheme.

\subsection{Basic Results in Linear Algebra and Multi-linear Representability}

In this section we give some basic results in linear algebra and matroid theory that are used in the paper. Recall that a matrix $A \in M_{n \times n}(\mathbb{F})$ is invertible if and only if it is of full rank if and only if $A \boldsymbol{v} \neq \mathbf{0}$ for every $\boldsymbol{v} \neq \mathbf{0}$. Also recall that block matrix multiplication can be carried out in block fashion, e.g., $\left[\begin{array}{ll}A & B \\ C & D\end{array}\right] \cdot\left[\begin{array}{ll}E & F \\ G & H\end{array}\right]=\left[\begin{array}{l}A E+B G A F+B H \\ C E+B G C F+D H\end{array}\right]$, as long as the dimensions match (note that the order written is important as usually $A E \neq E A$, etc.).

Proposition 2.11. Let $A, B, C$ be $k \times k$ matrices then

$$
\begin{aligned}
& \text { (a) } \operatorname{rank}\left[\begin{array}{ccc}
-I_{k} & 0 & C \\
A & -I_{k} & 0 \\
0 & B & -I_{k}
\end{array}\right]=2 k+\operatorname{rank}(B A C-I) . \\
& \text { (b) rank }\left[\begin{array}{cc}
-I_{k} & -I_{k} \\
A & B \\
0 & 0
\end{array}\right]=k+\operatorname{rank}(B-A) .
\end{aligned}
$$


Proof. Multiplying by invertible matrices does not change the rank of a matrix. Therefore,

$$
\begin{aligned}
\operatorname{rank}\left[\begin{array}{ccc}
-I_{k} & 0 & C \\
A & -I_{k} & 0 \\
0 & B & -I_{k}
\end{array}\right] & =\operatorname{rank}\left(\left[\begin{array}{ccc}
-I_{k} & 0 & C \\
A & -I_{k} & 0 \\
0 & B & -I_{k}
\end{array}\right] \cdot\left[\begin{array}{ccc}
I_{k} & 0 & C \\
0 & I_{k} & A \cdot C \\
0 & 0 & I_{k}
\end{array}\right]\right) \\
& =\operatorname{rank}\left[\begin{array}{ccc}
-I_{k} & 0 & 0 \\
A & -I_{k} & 0 \\
0 & B & B A C-I_{k}
\end{array}\right]=2 k+\operatorname{rank}\left(B A C-I_{k}\right) .
\end{aligned}
$$

and

$$
\begin{aligned}
\operatorname{rank}\left[\begin{array}{cc}
-I_{k} & -I_{k} \\
A & B \\
0 & 0
\end{array}\right] & =\operatorname{rank}\left(\left[\begin{array}{cc}
-I_{k} & -I_{k} \\
A & B \\
0 & 0
\end{array}\right] \cdot\left[\begin{array}{cc}
I_{k} & -I_{k} \\
0 & I_{k}
\end{array}\right]\right) \\
& =\operatorname{rank}\left[\begin{array}{cc}
-I_{k} & 0 \\
A & B-A \\
0 & 0
\end{array}\right]=k+\operatorname{rank}(B-A)
\end{aligned}
$$

Proposition 2.12. Let $\mathbf{B}:=\left[\begin{array}{ccc}B_{1,1} & \ldots & B_{1, n} \\ \vdots & \ddots & \vdots \\ B_{m, 1} & \ldots & B_{m, n}\end{array}\right]$ be a k-linear representation of a matroid $M$, with $B_{i, j}$ being $k \times k$ block matrices, and let $G$ be any invertible $k \times k$ matrix. Then:

a) For every $1 \leq i \leq n$ then $\left[\begin{array}{ccccc}B_{1,1} & \ldots & B_{1, j} \cdot G & \ldots & B_{1, n} \\ \vdots & \ddots & \vdots & \ddots & \vdots \\ B_{m, 1} & \ldots & B_{m, j} \cdot G & \ldots & B_{m, n}\end{array}\right]$ is a k-linear representation of $M$.

b) For every $1 \leq i \leq m$ then $\left[\begin{array}{ccc}B_{1,1} & \ldots & B_{1, n} \\ \vdots & \ddots & \vdots \\ G \cdot B_{i, 1} & \ldots & G \cdot B_{i, n} \\ \vdots & \ddots & \vdots \\ B_{m, 1} & \ldots & B_{m, n}\end{array}\right]$ is a k-linear representation of $M$.

c) If $\{1, \ldots, m\}$ is a base of $M$ then there exists a matrix of the form $\left[\begin{array}{cccccc}I_{k} & \ldots & 0 & B_{1, m+1}^{\prime} & \ldots & B_{1, n}^{\prime} \\ \vdots & \ddots & \vdots & \ddots & \vdots \\ 0 & \ldots & I_{k} & B_{m, m+1}^{\prime} & \ldots & B_{m, n}^{\prime}\end{array}\right]$ that is also a $k$-linear representation of $M$. 
Proof. a) Since $G$ is invertible, it is immediate from basic linear algebra that

$$
\begin{aligned}
& \operatorname{rank}\left[\begin{array}{ccccc}
B_{1, i_{1}} & \ldots & B_{1, i_{\ell}} & \ldots & B_{1, i_{s}} \\
\vdots & \ddots & \vdots & \ddots & \vdots \\
B_{m, i_{1}} & \ldots & B_{1, i_{\ell}} & \ldots & B_{m, i_{s}}
\end{array}\right] \\
& =\operatorname{rank}\left(\left[\begin{array}{ccccc}
B_{1, i_{1}} & \ldots & B_{1, i_{\ell}} & \ldots & B_{1, i_{s}} \\
\vdots & \ddots & \vdots & \ddots & \vdots \\
B_{m, i_{1}} & \ldots & B_{1, i_{\ell}} & \ldots & B_{m, i_{s}}
\end{array}\right] \cdot\left[\begin{array}{ccccc}
I_{k} & \ldots & 0 & \ldots & 0 \\
\vdots & \ddots & \vdots & \ddots & \vdots \\
0 & \ldots & G & \ldots & 0 \\
\vdots & \ddots & \vdots & \ddots & \vdots \\
0 & \ldots & 0 & \ldots & I_{k}
\end{array}\right]\right) \\
& =\operatorname{rank}\left[\begin{array}{ccccc}
B_{1, i_{1}} & \ldots & B_{1, i_{\ell}} \cdot G \ldots & \ldots & B_{1, i_{s}} \\
\vdots & \ddots & \vdots & \ddots & \vdots \\
B_{m, i_{1}} & \ldots & B_{1, i_{\ell}} \cdot G & \ldots & B_{m, i_{s}}
\end{array}\right] \text {, }
\end{aligned}
$$

for any submatrix (with $j=i_{\ell}$ ), which is exactly what we need to prove.

b) Simillarly, for any submatrix,

$$
\begin{gathered}
\left.\operatorname{rank}\left[\begin{array}{ccc}
B_{1,1} & \ldots & B_{1, n} \\
\vdots & \ddots & \vdots \\
B_{i, 1} & \ldots & B_{i, n} \\
\vdots & \ddots & \vdots \\
B_{m, 1} & \ldots & B_{m, n}
\end{array}\right]=\operatorname{rank}\left[\begin{array}{ccccc}
I_{k} & \ldots & 0 & \ldots & 0 \\
\vdots & \ddots & \vdots & \ddots & \vdots \\
0 & \ldots & G & \ldots & 0 \\
\vdots & \ddots & \vdots & \ddots & \vdots \\
0 & \ldots & 0 & \ldots & I_{k}
\end{array}\right] \cdot\left[\begin{array}{ccc}
B_{1,1} & \ldots & B_{1, n} \\
\vdots & \ddots & \vdots \\
B_{i, 1} & \ldots & B_{i, n} \\
\vdots & \ddots & \vdots \\
B_{m, 1} & \ldots & B_{m, n}
\end{array}\right]\right) \\
=\operatorname{rank}\left[\begin{array}{ccc}
B_{1,1} & \ldots & B_{1, n} \\
\vdots & \ddots & \vdots \\
G \cdot B_{i, 1} & \ldots & G \cdot B_{i, n} \\
\vdots & \ddots & \vdots \\
B_{m, 1} & \ldots & B_{m, n}
\end{array}\right] .
\end{gathered}
$$

c) Since $\{1, \ldots, \mathrm{m}\}$ is a base of $M$ then the columns $c_{1}, \ldots, c_{m \cdot k}$ of $\mathbf{B}$ are a basis of the column space of $\mathbf{B}$ (which is, therefore, $\mathbb{F}^{k \cdot m}$ ). Therefore, there is an invertible linear transformation $T$ such that $\forall 1 \leq i \leq m k, T\left(c_{i}\right)=e_{i}$. Since $T$ is invertible $\operatorname{dim}\left(\operatorname{span}\left\{T\left(c_{i_{1}}\right), \ldots, T\left(c_{i_{j}}\right)\right\}\right)=\operatorname{dim}\left(\operatorname{span}\left\{c_{i_{1}}, \ldots, c_{i_{j}}\right\}\right)$ for any set of columns $\left\{c_{i_{1}}, \ldots, c_{i_{j}}\right\}$, which implies that by applying $T$ to all the columns of $\mathbf{B}$ we get that

$$
\left[\begin{array}{ccccccc}
I_{k} & 0 & \ldots & 0 & \vdots & \ldots & \vdots \\
0 & I_{k} & \ldots & 0 & T\left(c_{k m+1}\right) & \ldots & T\left(c_{k n}\right) \\
\vdots & \vdots & \ddots & \vdots & \vdots & & \vdots \\
0 & 0 & \ldots & I_{k} & \cdot & & \cdot
\end{array}\right]
$$

is a $k$-linear representation of $M$ 
We will call operations Proposition 2.12( $(a)$ and 2.12(b) column and row blockscaling respectively.

\subsection{Fixed-Point Free Representations}

A standard tool in studying groups is representation theory. Our result relies heavily on theorems from this extensively researched field of mathematics. We will only give the necessary definitions and state the result. We then sketch the main ideas of the proof of this result. The complete proof, which requires much more representation theory, will appear in the full version.

Definition 2.13. Let $G$ be a finite group and $\mathbb{F}$ a field. A representation of $G$ is a group homomorphism $\rho: G \rightarrow \mathrm{GL}_{n}(\mathbb{F})$ (the group of $n \times n$ invertible matrices). The dimension or degree of a representation is $n$. A representation is called faithful if it is injective. A representation $\rho: G \rightarrow \mathrm{GL}_{n}(\mathbb{F})$ is fixed-point free if for every $1 \neq g \in G$ the field element 1 is not an eigenvalue of $\rho(g)$, i.e., $\rho(g) \cdot v \neq v$ for every $g \neq 1$ and for every $v \neq 0$. A fixed-point free group is one which has a fixed-point free representation.

We note that not all representations of a fixed-point free group $G$ are fixedpoint free, even if the representation is faithful. For example, cyclic groups are fixed-point free, but also admit non fixed-point free representations:

Example 2.14. Let $G=\mathbb{Z}_{m}$ be the additive group with $m$ elements. Denote $\zeta=$ $e^{\frac{2 \pi i}{m}}$. If $\rho: G \rightarrow \mathrm{GL}_{2}(\mathbb{C})$ is defined by $\rho(k)=\left(\begin{array}{cc}\zeta^{k} & 0 \\ 0 & 1\end{array}\right)$ then $\rho$ is faithful (because $i \neq k \Rightarrow \rho(i) \neq \rho(k))$ but not fixed-point free because $\left(\begin{array}{ll}\zeta^{k} & 0 \\ 0 & 1\end{array}\right)\left(\begin{array}{l}0 \\ 1\end{array}\right)=\left(\begin{array}{l}0 \\ 1\end{array}\right)$ (and this should only happen for $k=0)$. However, if we define $\rho(k)=\left(\begin{array}{cc}\zeta^{k} & 0 \\ 0 & \zeta^{k}\end{array}\right)$ then $\rho$ is fixed-point free, because if $k \neq 0$ then 1 is not an eigenvalue of $\left(\begin{array}{cc}\zeta^{k} & 0 \\ 0 & \zeta^{k}\end{array}\right)$. We note that the group $\mathbb{Z}_{m}$ also has a fixed-point free representation of dimension 1 , by $\rho(k)=\left(e^{\frac{2 k \pi i}{m}}\right)$.

Fixed-point free groups have been completely classified by the works of Burnside and later Vincent [40] and Zassenhaus [44. The classification can be found, for example, in [42. For our purposes we will require only the following result, easily achieved from the classification:

Proposition 2.15. For every prime $p>2$, there exist a prime $q>p$ and $a$ group $G_{p}$ of order $p^{2} q$ such that:

1. $G_{p}$ has a fixed-point free representation of dimension $p$ over the field $\mathbb{F}_{2^{p q}}$, i.e., the field of characteristic 2 with $2^{p q}$ elements.

2. The group $G_{p}$ does not admit a fixed-point representation of dimension less than $p$ over any field. 
Moreover, there exists such $q$ with $q=O\left(p^{5.18}\right)$, so the field $\mathbb{F}_{2^{p q}}$ has $2^{O\left(p^{6.18}\right)}$ elements.

Our proof uses the construction of semidirect product of groups. The definition can be found in most group theory books. See for example [26]. It also requires some classical theorems from representation theory, which can be found, for example, in 31 .

The complete proof of Proposition 2.15 will be given in the full version. We now sketch the main ideas of the proof.

Proof Sketch. Let $p>2$ be a prime number. From Linnik's Theorem [22 23], there exists a prime $q$ such that $q=n p+1$ for some $n \in \mathbb{N}$, and $q$ is polynomially bounded by $p$. The state of the art improvement, by Xylouris [43], shows that $q=O\left(p^{5.18}\right)$.

From the fact that $q=n p+1$, it can be deduced that there exists a nontrivial semidirect product $G_{p}=\mathbb{Z}_{q} \rtimes \mathbb{Z}_{p^{2}}$, with the action of $\mathbb{Z}_{p}$ (we give a brief explanation of the construction of this group, and why this group works, in Appendix A]. We then show, both directly and using the classification of fixed-point free groups, that the group $G_{p}$ is fixed-point free, and, thus, has a fixed-point free representation.

Then, using classical theorems from representation theory, we show that a fixed-point free representation of $G_{p}$ is of dimension at least $p$, and that there indeed exists a fixed-point free representation of dimension $p$. In particular, we show directly that there exists such a representation over the field $\mathbb{F}_{2^{p q}}$, which has $2^{p q}=2^{O\left(p^{6.18}\right)}$ elements.

\section{Main Theorem and Result}

In this section, we prove that there is an access structure that has an ideal $p$ linear secret-sharing scheme and does not have an ideal $k$-linear secret-sharing scheme for every $k<p$. As explained in Section 2.3, it suffices to prove that there is a matroid that is $p$-minimally representable. We prove this result for the Dowling matroid, for an appropriate group $G$. We next state our main theorem.

Theorem 3.1. For a finite group $G$, the matroid $Q_{3}(G)$ is $k$-linearly representable over a field $\mathbb{F}$ if and only if there is a fixed-point free representation $\rho: G \rightarrow \mathrm{GL}_{k}(\mathbb{F})$.

The main contribution of the theorem is the new connection between multi-linear representation of the Dowling matroid over $G$ to the existence of a fixed-point free representation of the group $G$. The theorem transfers the problem of multilinear representablity of $Q_{3}(G)$ to finding fixed-point free representations of $G$. Since fixed-point free groups and representations have been completely classified, it gives a complete answer to this problem.

To discuss the representations of $Q_{3}(G)$, we define the following block matrix $\mathbf{A}_{\rho}$. In Lemma [3.2, we will prove that if $Q_{3}(G)$ is multi-linearly representable, 
then $\mathbf{A}_{\rho}$ is a multi-linear representation of $Q_{3}(G)$ for some representation $\rho$ of $G$. Then we prove in Lemmas 3.3 and 3.4 that $\mathbf{A}_{\rho}$ represents $Q_{3}(G)$ if and only if $\rho$ is fixed-point free.

For a finite group $G=\left\{1=g_{1}, g_{2}, \ldots, g_{n}\right\}$, a field $\mathbb{F}$, and a faithful representation $\rho: G \rightarrow \mathrm{GL}_{k}(\mathbb{F})$ we denote by $\mathbf{A}_{\rho}$ the following block matrix, which contains $3 k$ rows and $3(n+1) k$ columns.

$$
\mathbf{A}_{\rho}:=\left[\begin{array}{cccccccccccc}
I_{k} & 0 & 0 & -I_{k} & \ldots & -I_{k} & 0 & \ldots & 0 & \rho\left(g_{1}\right) & \ldots & \rho\left(g_{n}\right) \\
0 & I_{k} & 0 & \rho\left(g_{1}\right) & \ldots & \rho\left(g_{n}\right) & -I_{k} & \ldots & -I_{k} & 0 & \ldots & 0 \\
0 & 0 & I_{k} & 0 & \ldots & 0 & \rho\left(g_{1}\right) & \ldots & \rho\left(g_{n}\right) & -I_{k} & \ldots & -I_{k}
\end{array}\right]
$$

Lemma 3.2. If $M=Q_{3}(G)$ is $k$-linearly representable over $\mathbb{F}$, then there exists a faithful representation $\rho: G \rightarrow \mathrm{GL}_{k}(\mathbb{F})$ such that $\mathbf{A}_{\rho}$ is a $k$-linear representation of $M$.

Proof. The technique we use to prove this lemma is a standard one (e.g., see the proofs of [28, Proposition 6.4.8, Lemma 6.8.5, Theorem 6.10.10] and [29, Lemma $3.35]$ ). We generalize this technique to multi-linear representations by looking at the representation matrix as a block matrix and using Proposition 2.12. We repeatedly use the fact that for any multi-linear representation of $M$, if $X \subseteq E$ and $r(X)=n$ then the rank of the relevant sub-matrix of the representation (i.e., deleting the columns of elements not in $X$ ) is $n \cdot k$.

Suppose that

$$
\mathbf{B}:=\left[\begin{array}{lllllllllll}
B_{1,1} & B_{1,2} & B_{1,3} & B_{1, g_{1}^{\prime}} \ldots & B_{1, g_{n}^{\prime}} & B_{1, g_{1}^{\prime \prime}} \ldots & B_{1, g_{n}^{\prime \prime}} & B_{1, g_{1}^{\prime \prime \prime}} \ldots & B_{1, g_{n}^{\prime \prime \prime}} \\
B_{2,1} & B_{2,2} & B_{2,3} & B_{2, g_{1}^{\prime}} \ldots & B_{2, g_{n}^{\prime}} & B_{2, g_{1}^{\prime \prime}} \ldots & B_{2, g_{n}^{\prime \prime}} & B_{2, g_{1}^{\prime \prime \prime}} \ldots & B_{2, g_{n}^{\prime \prime \prime}} \\
B_{3,1} & B_{3,2} & B_{3,3} & B_{3, g_{1}^{\prime}} \ldots & B_{3, g_{n}^{\prime}} & B_{3, g_{1}^{\prime \prime}} \ldots & B_{3, g_{n}^{\prime \prime}} & B_{3, g_{1}^{\prime \prime \prime}} \ldots & B_{3, g_{n}^{\prime \prime \prime}}
\end{array}\right]
$$

is a $k$-linear representation of $M$. Then $r(\{1,2,3\})=3=r(M)$ so $\mathbf{B}_{1}, \ldots, \mathbf{B}_{3 k}$ span the columns of $\mathbf{B}$. By changing the basis of the column space of $\mathbf{B}$ (see Proposition $2.12(c)$ ) there exists a block matrix $\mathbf{C}$ of the form

$$
\mathbf{C}:=\left[\begin{array}{cccccccccc}
I_{k} & 0 & 0 & C_{1, g_{1}^{\prime}} \ldots & C_{1, g_{n}^{\prime}} & C_{1, g_{1}^{\prime \prime}} \ldots & C_{1, g_{n}^{\prime \prime}} & C_{1, g_{1}^{\prime \prime \prime}} \ldots C_{1, g_{n}^{\prime \prime \prime}} \\
0 & I_{k} & 0 & C_{2, g_{1}^{\prime}} \ldots & C_{1, g_{n}^{\prime}} & C_{2, g_{1}^{\prime \prime}} \ldots C_{2, g_{n}^{\prime \prime}} & C_{2, g_{1}^{\prime \prime \prime}} \ldots C_{2, g_{n}^{\prime \prime \prime}} \\
0 & 0 & I_{k} & C_{3, g_{1}^{\prime}} \ldots & \ldots C_{1, g_{n}^{\prime}} & C_{3, g_{1}^{\prime \prime}} \ldots C_{3, g_{n}^{\prime \prime}} & C_{3, g_{1}^{\prime \prime \prime}} \ldots C_{3, g_{n}^{\prime \prime \prime}}
\end{array}\right]
$$

that is a $k$-linear representation of $M$. As $\forall g \in G, r\left(\left\{1,2, g^{\prime}\right\}\right)=2$, we have that

$$
\operatorname{rank}\left[\begin{array}{ccc}
I_{k} & 0 & C_{1, g^{\prime}} \\
0 & I_{k} & C_{2, g^{\prime}} \\
0 & 0 & C_{3, g^{\prime}}
\end{array}\right]=2 k
$$

Thus, $C_{3, g^{\prime}}=0$. Also $r\left(\left\{1, g^{\prime}\right\}\right)=2$, so

$$
\operatorname{rank}\left[\begin{array}{cc}
I_{k} & C_{1, g^{\prime}} \\
0 & C_{2, g^{\prime}} \\
0 & C_{3, g^{\prime}}
\end{array}\right]=2 k
$$


therefore, $C_{2, g^{\prime}}$ is invertible (it has to be of full rank since $C_{3, g^{\prime}}=0$ ). Since $r\left(\left\{2, g^{\prime}\right\}\right)=2$, by the same argument $C_{1, g^{\prime}}$ is also invertible. Similarly $\forall g \in G$, $C_{1, g^{\prime \prime}}=0$, and $C_{3, g^{\prime \prime}}, C_{2, g^{\prime \prime}}$ are invertible, and $C_{2, g^{\prime \prime \prime}}=0$, and $C_{1, g^{\prime \prime \prime}}, C_{3, g^{\prime \prime \prime}}$ are invertible.

We now apply column block-scaling (Proposition 2.12 $(a)$ ) on the columns of $g_{1}^{\prime}, \ldots, g_{n}^{\prime}$ by $-\left(C_{1, g_{1}^{\prime}}\right)^{-1}, \ldots,-\left(C_{1, g_{n}^{\prime}}\right)^{-1}$ respectively to get that

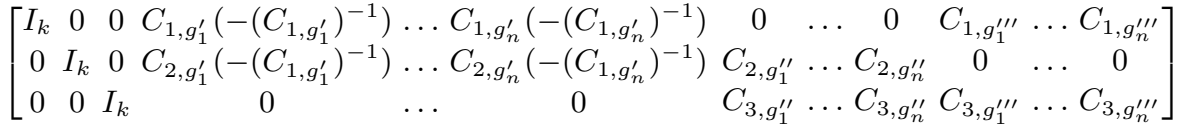

$$
\begin{aligned}
& =\left[\begin{array}{cccccccccccc}
I_{k} & 0 & 0 & -I_{k} & \ldots & -I_{k} & 0 & \ldots & 0 & C_{1, g_{1}^{\prime \prime}} & \ldots & C_{1, g_{n}^{\prime \prime \prime}} \\
0 & I_{k} & 0 & C_{2, g_{1}^{\prime}}^{\prime} & \ldots & C_{2, g_{n}^{\prime}}^{\prime} & C_{2, g_{1}^{\prime \prime}} & \ldots & C_{2, g_{n}^{\prime \prime}} & 0 & \ldots & 0 \\
0 & 0 & I_{k} & 0 & \ldots & 0 & C_{3, g_{1}^{\prime \prime}} & \ldots & C_{3, g_{n}^{\prime \prime}} & C_{3, g_{1}^{\prime \prime}} & \ldots & C_{3, g_{n}^{\prime \prime \prime}}
\end{array}\right]
\end{aligned}
$$

is a $k$-linear representation of $M$. Now by row block-scaling (Propostion 2.12 $(b)$ ) on the second row by $\left(C_{2, g_{1}^{\prime}}^{\prime}\right)^{-1}$ we get that

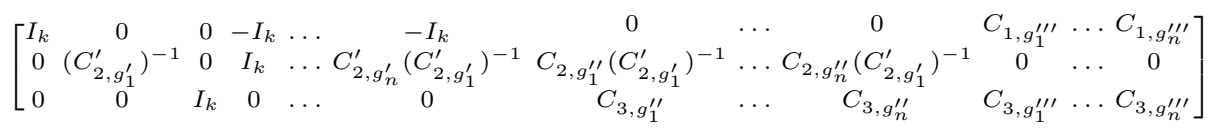

is a $k$-linear representation of $M$. We continue in the same fashion by blockscaling on the columns of $g_{1}^{\prime \prime}, \ldots, g_{n}^{\prime \prime}$, then row block-scaling on the third row, then column block-scaling of columns $g_{1}^{\prime \prime \prime}, \ldots, g_{n}^{\prime \prime \prime}$, and finally column block scaling of columns 2,3 to get that

$$
\mathbf{D}:=\left[\begin{array}{ccccccccccccccc}
I_{k} & 0 & 0 & -I_{k} & -I_{k} & \ldots & -I_{k} & 0 & 0 & \ldots & 0 & D_{1, g_{1}^{\prime \prime \prime}} & D_{1, g_{2}^{\prime \prime \prime}} & \ldots & D_{1, g_{n}^{\prime \prime \prime}} \\
0 & I_{k} & 0 & I_{k} & D_{2, g_{2}^{\prime}} & \ldots & D_{2, g_{n}^{\prime}} & -I_{k} & -I_{k} & \ldots & -I_{k} & 0 & 0 & \ldots & 0 \\
0 & 0 & I_{k} & 0 & 0 & \ldots & 0 & I_{k} & D_{3, g_{2}^{\prime \prime}} & \ldots & D_{3, g_{n}^{\prime \prime}} & -I_{k} & -I_{k} & \ldots & -I_{k}
\end{array}\right]
$$

is a $k$-linear representation of $M$.

We next use the fact that $\mathbf{D}$ is a multi-linear representation of $Q_{3}(G)$ to prove that blocks in different parts of the representation are equal, e.g., $D_{3, g^{\prime \prime}}=D_{2, g^{\prime}}$. Since $r\left(\left\{g_{1}^{\prime}, g_{1}^{\prime \prime}, g_{1}^{\prime \prime \prime}\right\}\right)=2$, we have that

$$
\operatorname{rank}\left[\begin{array}{ccc}
-I_{k} & 0 & D_{1, g_{1}^{\prime \prime \prime}} \\
I_{k} & -I_{k} & 0 \\
0 & I_{k} & -I_{k}
\end{array}\right]=2 k
$$

and this forces $D_{1, g_{1}^{\prime \prime \prime}}=I_{k}$. For $j, \ell$ such that $g_{j}=g_{\ell}^{-1}$ (thus, $g_{j} \cdot g_{1} \cdot g_{\ell}=1$ ), we have that $r\left(\left\{g_{1}^{\prime}, g_{j}^{\prime \prime}, g_{\ell}^{\prime \prime \prime}\right\}\right)=2$. So,

$$
\operatorname{rank}\left[\begin{array}{ccc}
-I_{k} & 0 & D_{1, g_{\ell}^{\prime \prime \prime}} \\
I_{k} & -I_{k} & 0 \\
0 & D_{3, g_{j}^{\prime \prime}} & -I_{k}
\end{array}\right]=2 k
$$

By Proposition 2.11 $(a)$ we get that $\operatorname{rank}\left(D_{3, g_{j}^{\prime \prime}} \cdot D_{1, g_{\ell}^{\prime \prime \prime}}-I_{k}\right)=0$ so $D_{3, g_{j}^{\prime \prime}}=$ $\left(D_{1, g_{\ell}^{\prime \prime \prime}}\right)^{-1}$. By symmetric arguments, $D_{1, g_{j}^{\prime \prime \prime}}=\left(D_{2, g_{\ell}^{\prime}}\right)^{-1}$ and $D_{2, g_{j}^{\prime}}=\left(D_{3, g_{\ell}^{\prime \prime}}\right)^{-1}$. 
Therefore,

$$
\forall g \in G, D_{3, g^{\prime \prime}}=D_{2, g^{\prime}}=D_{1, g^{\prime \prime \prime}} .
$$

Now let $\rho: G \rightarrow \mathrm{GL}_{k}(\mathbb{F})$ be the map $\rho(g)=D_{2, g^{\prime}}$. We see that $\rho(1)=I$ (because $\left.D_{2, g_{1}^{\prime}}=I\right)$. By Proposition 2.11( $(a)$

$$
\begin{aligned}
\operatorname{rank}\left[\begin{array}{ccc}
-I_{k} & 0 & D_{1, g_{\ell}^{\prime \prime \prime}} \\
D_{2, g_{i}^{\prime}} & -I_{k} & 0 \\
0 & D_{3, g_{j}^{\prime \prime}} & -I_{k}
\end{array}\right] & =2 k+\operatorname{rank}\left(D_{3, g_{j}^{\prime \prime}} D_{2, g_{i}^{\prime}} D_{1, g_{\ell}^{\prime \prime \prime}}-I\right) \\
& =2 k+\operatorname{rank}\left(\rho\left(g_{j}\right) \cdot \rho\left(g_{i}\right) \cdot \rho\left(g_{\ell}\right)-I\right) .
\end{aligned}
$$

By the matroid rank, it is equal to $2 k$ if $g_{j} \cdot g_{i} \cdot g_{\ell}=1$ and $3 k$ otherwise, thus,

$$
\forall g_{i}, g_{j}, g_{\ell} \in G, g_{j} \cdot g_{i} \cdot g_{\ell}=1 \Leftrightarrow \rho\left(g_{j}\right) \cdot \rho\left(g_{i}\right) \cdot \rho\left(g_{\ell}\right)=I .
$$

We now use (5) to show that $\rho$ is an injective group homomorphism, which completes the proof:

For every $g \in G$, since $1 \cdot g^{-1} \cdot g=1$, we have $I=\rho(1) \cdot \rho\left(g^{-1}\right) \cdot \rho(g)=$ $I \cdot \rho\left(g^{-1}\right) \cdot \rho(g)$, forcing $\rho(g)^{-1}=\rho\left(g^{-1}\right)$.

Therefore, $\forall g, h \in G$, as $g \cdot h \cdot(g h)^{-1}=1$, we have $I=\rho(g) \cdot \rho(h) \cdot \rho\left((g h)^{-1}\right)=$ $\rho(g) \cdot \rho(h) \cdot \rho(g h)^{-1}$. Thus, $\rho(g h)=\rho(g) \cdot \rho(h)$. This proves that $\rho$ is a group homomorphism.

For injectivity, if $g \neq h$ then $g \cdot h^{-1} \cdot 1 \neq 1$, which implies that $\rho(g) \cdot \rho(h)^{-1}$. $\rho(1) \neq I$, so $\rho(g) \neq \rho(h)$.

Lemma 3.3. Let $\rho: G \rightarrow \mathrm{GL}_{k}(\mathbb{F})$ be a faithful representation. If $\mathbf{A}_{\rho}$ is a $k$ linear representation of $Q_{3}(G)$ then $\rho$ is fixed-point free.

Proof. Since $\mathbf{A}_{\rho}$ is a $k$-linear representation of $Q_{3}(G)$, for every $g \neq 1_{G}$ we have that $r\left(\left\{g_{1}^{\prime}, g^{\prime}\right\}\right)=2$. So

$$
\operatorname{rank}\left[\begin{array}{cc}
-I_{k} & -I_{k} \\
I_{k} & \rho(g) \\
0 & 0
\end{array}\right]=2 k
$$

By Proposition 2.11 (b) we have that

$$
\operatorname{rank}\left[\begin{array}{cc}
-I_{k} & -I_{k} \\
I_{k} & \rho(g) \\
0 & 0
\end{array}\right]=k+\operatorname{rank}\left(\rho(g)-I_{k}\right)
$$

By combining (6) and (7), $\operatorname{rank}\left(\rho(g)-I_{k}\right)=k$. This implies that $\rho(g)-I_{k}$ is invertible, so $\forall v \neq 0,\left(\rho(g)-I_{k}\right) v \neq 0$, therefore, $\forall v \neq 0, \rho(g) v \neq v$, which means that 1 is not an eigenvalue of $\rho(g)$. So, $\rho$ is fixed-point free, as desired.

Lemma 3.4. If $\rho: G \rightarrow \mathrm{GL}_{k}(\mathbb{F})$ is a fixed-point free representation, then $\mathbf{A}_{\rho}$ is a $k$-linear representation of $Q_{3}(G)$. 
Proof. To prove that $\mathbf{A}_{\rho}$ is a $k$-linear representation of $M$, we need to verify that $\forall X \subset E$, if $r(X)=n$ then the rank of the relevant sub-matrix of $\mathbf{A}_{\rho}$ (i.e., deleting the columns of elements not in $X$ ) is $n k$. Ranks of most sub-matrices are trivially verified, e.g.,

$$
\operatorname{rank}\left[\begin{array}{ccc}
I_{k} & 0 & -I_{k} \\
0 & I_{k} & 0 \\
0 & 0 & \rho(g)
\end{array}\right]=\operatorname{rank}\left[\begin{array}{ccc}
I_{k} & -I_{k} & -I_{k} \\
0 & \rho\left(g_{i}\right) & 0 \\
0 & 0 & \rho\left(g_{j}\right)
\end{array}\right]=3 k, \operatorname{rank}\left[\begin{array}{cc}
I_{k} & -I_{k} \\
0 & \rho(g) \\
0 & 0
\end{array}\right]=2 k \text {. }
$$

(Note that $\forall g \in G$, the matrix $\rho(g)$ is invertible, and, therefore, of rank $k$ ). So it is necessary and sufficient to ensure that the following 2 requirements hold:

1. For every two distinct elements $g_{i} \neq g_{j}$

$$
\operatorname{rank}\left[\begin{array}{cc}
-I_{k} & -I_{k} \\
\rho\left(g_{i}\right) & \rho\left(g_{j}\right) \\
0 & 0
\end{array}\right]=2 k
$$

2. For all $g_{i}, g_{j}, g_{\ell} \in G$ (not necessarily distinct)

$$
\operatorname{rank}\left[\begin{array}{ccc}
-I_{k} & 0 & \rho\left(g_{\ell}\right) \\
\rho\left(g_{i}\right) & -I_{k} & 0 \\
0 & \rho\left(g_{j}\right) & -I_{k}
\end{array}\right]=r\left(\left\{g_{i}^{\prime}, g_{j}^{\prime \prime}, g_{\ell}^{\prime \prime \prime}\right\}\right)=\left\{\begin{array}{cc}
2 k & \text { if } g_{j} \cdot g_{i} \cdot g_{\ell}=1 \\
3 k & \text { otherwise }
\end{array}\right.
$$

Ranks of all other relevant sub-matrices follow from similar arguments.

We first show that Equation (8) holds. By Proposition 2.11(b)

$$
\operatorname{rank}\left[\begin{array}{cc}
-I_{k} & -I_{k} \\
\rho\left(g_{i}\right) & \rho\left(g_{j}\right) \\
0 & 0
\end{array}\right]=k+\operatorname{rank}\left(\rho\left(g_{j}\right)-\rho\left(g_{i}\right)\right)
$$

so in order to show that Equation (8) holds, we need to verify that for every two distinct group elements $g_{i}, g_{j} \operatorname{rank}\left(\rho\left(g_{i}\right)-\rho\left(g_{j}\right)\right)=k$. Since $\rho$ is fixedpoint free and $g_{i}^{-1} g_{j} \neq 1$, for every $v \neq 0, v \neq \rho\left(g_{i}^{-1} g_{j}\right) v=\left(\rho\left(g_{i}\right)^{-1} \rho\left(g_{j}\right)\right) v$, so $\forall v \neq 0, \rho\left(g_{i}\right) v \neq \rho\left(g_{j}\right) v$, thus, $\forall v \neq 0,\left(\rho\left(g_{i}\right)-\rho\left(g_{j}\right)\right) v \neq 0$, which implies that $\rho\left(g_{i}\right)-\rho\left(g_{j}\right)$ is invertible and, therefore, of rank $k$, so (8) holds.

We next show that Equation (9) holds. By Proposition 2.11 (a) and the definition of a homomorphism,

$$
\operatorname{rank}\left[\begin{array}{ccc}
-I_{k} & 0 & \rho\left(g_{\ell}\right) \\
\rho\left(g_{i}\right) & -I_{k} & 0 \\
0 & \rho\left(g_{j}\right) & -I_{k}
\end{array}\right]=2 k+\operatorname{rank}\left(\rho\left(g_{j} \cdot g_{i} \cdot g_{\ell}\right)-I_{k}\right) .
$$

So, to prove that (9) holds, we need to show that

$$
\operatorname{rank}\left(\rho\left(g_{j} \cdot g_{i} \cdot g_{\ell}\right)-I_{k}\right)= \begin{cases}0 \text { if } g_{j} \cdot g_{i} \cdot g_{\ell}=1 \\ k & \text { otherwise }\end{cases}
$$

By arguments similar to the above 
1. If $g_{j} \cdot g_{i} \cdot g_{\ell} \neq 1$ then $\operatorname{rank}\left(\rho\left(g_{j} \cdot g_{i} \cdot g_{\ell}\right)-I_{k}\right)=k$, as $\rho$ is fixed-point free.

2. If $g_{j} \cdot g_{i} \cdot g_{\ell}=1$ then $\operatorname{rank}\left(\rho\left(g_{j} \cdot g_{i} \cdot g_{\ell}\right)-I_{k}\right)=0$. (This in fact true for any representation because $\rho\left(g_{j} \cdot g_{i} \cdot g_{\ell}\right)=\rho(1)=I_{k}$.)

Proof (Proof of Theorem 3.1). Combining the lemmas we get Theorem 3.1 If $G$ has a fixed-point free representation $\rho$ of dimension $k$, then by Lemma 3.4 the block matrix $\mathbf{A}_{\rho}$ is a $k$-linear representation of $Q_{3}(G)$, and, in particular, $Q_{3}(G)$ has a $k$-linear representation. On the other hand, if $Q_{3}(G)$ is $k$-linearly representable then, by Lemma 3.2 it has a faithful representation $\rho$ of dimension $k$ such that $\mathbf{A}_{\rho}$ is a $k$-linear representation of $Q_{3}(G)$, so, by Lemma $3.3 \rho$ is fixed-point free.

We combine Theorem 3.1 with Proposition 2.15 to get our desired result:

Corollary 3.5. For every prime $p>2$ there is a matroid that is $p$-minimally representable. Moreover, the matroid has poly $(p)$ ground points and this representation exists over a finite field with $2^{O\left(p^{6.18}\right)}$ elements.

Proof. Let $q$ and $G_{p}$ be as in Proposition 2.15. By Theorem 3.1] and Proposition 2.15 over the field $\mathbb{F}_{2^{p q}}$, the matroid $Q_{3}\left(G_{p}\right)$, which has $3 p^{2} q+3$ elements in the ground set, is $p$-linearly representable. Furthermore, over any field, the matroid $Q_{3}\left(G_{p}\right)$ is not $j$-linearly representable for any $j<p$. So, $Q_{3}\left(G_{p}\right)$ is $p$-minimally representable. By Proposition 2.15, if we chose the appropriate $q$, then the field $\mathbb{F}_{2^{p q}}$ has $2^{O\left(p^{6.18}\right)}$ elements.

We next rephrase the result in secret-sharing terms.

Corollary 3.6. For every prime $p$, there exists an access structure with $\operatorname{poly}(p)$ parties, which has an ideal p-linear secret-sharing scheme with secrets of length poly $(n)$, but has no ideal $k$-linear secret-sharing scheme for every $k<p$.

Since the matroid has $3 p^{2} q+3$ elements in the ground set, the corresponding access structure has $3 p^{2} q+2$ parties. Therefore, for every prime $p$, the smallest access structure of this type has $O\left(p^{7.18}\right)$ parties. Also note that the schemes is over a field with $2^{\text {poly }(p)}$ elements, so every share can be represented by $\operatorname{poly}(p)$ bits.

\section{Lower Bounds for Multi-linear Secret-Sharing Schemes}

The best known lower bounds for linear secret-sharing schemes is $n^{\Omega(\log n)}[11617$. By modification of the claims in [17, we show that these lower bounds hold also for multi-linear secret-sharing schemes. Thus, even using multi-linear schemes one cannot construct efficient schemes for general access structures.

We will use the following alternative definition of multi-linear secret sharing schemes, proven to be equivalent in [13] (following [7|20]). 
Definition 4.1 (Multi-Target Monotone Span Program). A multi-target monotone span program is a quadruple $\mathcal{M}=(\mathbb{F}, M, \rho, X)$, where $\mathbb{F}$ is a finite field, $M$ is an $a \times b$ matrix over $\mathbb{F}$, the function $\rho:\{1, \ldots, a\} \rightarrow\left\{p_{1}, \ldots, p_{n}\right\}$ labels each row of $M$ by a party, and $X$ is a set of $k$ independent vectors in $\mathbb{F}^{b}$ such that for every $A \subseteq\left\{p_{1}, \ldots, p_{n}\right\}$ either

- The rows of the sub-matrix obtained by restricting $M$ to the rows labeled by parties in $A$, denoted $M_{A}$, span every vector in $X$. In this case, we say that $\mathcal{M}$ accepts $A$, or,

- The rows of $M_{A}$ span no non-zero vector in the linear space spanned by $X$. In this case, we say that $\mathcal{M}$ rejects $B$.

We say that $\mathcal{M}$ accepts an access structure $\mathcal{A}$ if $\mathcal{M}$ accepts a set $B$ if $B \in \mathcal{A}$, and rejects every set $B \notin \mathcal{A}$. The size of a multi-target monotone span program is $a / k$, where $a$ is the number of rows in the matrix and $k$ is the number of vectors in the set $X$.

Note that not every labeled matrix is a multi-target span program. For example, if $k>1$ and for some set $A$, the rows in $M_{A}$ span exactly one vector in $X$, then this is not a multi-target span program. By [13] a multi-linear secret-sharing scheme realizing an access structure $\mathcal{A}$ with total share size $a$ exists if and only if there exists a multi-target monotone span program accepting $\mathcal{A}$ that has $a$ rows. In particular, if there exists a multi-target monotone span program accepting $\mathcal{A}$ with $a_{j}$ rows labeled by $p_{j}$ for $1 \leq j \leq n$ and $k$ vectors in the set $X$, then the exists a multi-linear secret-sharing scheme realizing $\mathcal{A}$ with information ratio $\max _{1 \leq j \leq n} a_{j} / k$. In ideal multi-linear secret-sharing schemes $a_{j}=k$ for every $j$.

Assume, w.l.o.g., that $X=\left\{\boldsymbol{e}_{1}, \ldots, \boldsymbol{e}_{k}\right\}$. We make 2 observations regarding multi-target monotone span program.

Observation 4.2. If $B \in \mathcal{A}$ and $N=M_{B}$ then the rows of $N$ span $X$, thus $\forall 0<s<k$ there exists some vector $\boldsymbol{v}_{s}$ such that $\boldsymbol{e}_{s}=\boldsymbol{v}_{s} N$.

Observation 4.3. If $T \notin \mathcal{A}$ then for every $s \in\{1, \ldots, k\}$ there exists a vector $\boldsymbol{w}_{s} \in \mathbb{F}^{b}$ such that the following hold: (1) $M_{T} \boldsymbol{w}_{s}=0$, (2) $\forall i \neq s, \boldsymbol{e}_{i} \cdot \boldsymbol{w}_{s}=0$, and (3) $\boldsymbol{e}_{s} \cdot \boldsymbol{w}_{s}=1$ (that is, the coordinate $s$ in $\boldsymbol{w}_{s}$ is 1 ).

Proof. If $T \notin \mathcal{A}$, then the rows of $M_{T}$ do not span any of the vectors in $X$. Let $M_{T, X}$ be the matrix containing the rows of $M_{T}$ and additional rows $\boldsymbol{e}_{1}, \ldots, \boldsymbol{e}_{k}$ and $M_{T, X \backslash\{s\}}$ the same matrix with the row $\boldsymbol{e}_{s}$ deleted. By simple linear algebra, for every $1 \leq s \leq k$, we have that $\operatorname{rank} M_{T, X}>\operatorname{rank} M_{T, X \backslash\{s\}}$, which implies that $\mid$ kernel $M_{T, X}|<|$ kernel $M_{T, X \backslash\{s\}} \mid$, and so there is some vector $\boldsymbol{w}_{s} \in \mathbb{F}^{b}$ such that $\boldsymbol{e}_{s} \cdot \boldsymbol{w}_{s}=1$ and $M_{T, X \backslash\{s\}} \boldsymbol{w}_{s}=\mathbf{0}$ (so evidently $M_{T} \boldsymbol{w}_{s}=0$ and $\left.\forall i \neq s, \boldsymbol{e}_{i} \cdot \boldsymbol{w}_{s}=0\right)$.

We next quote the definition of a collection with unique intersection from [17]. Such collections are used in [17] to prove lower bounds for monotone span programs; we show that the same lower bound holds for multi-target monotone span programs. 
Definition 4.4. Let $\mathcal{A}$ be a monotone access structure, with $\mathcal{B}=\left\{B_{1}, \ldots, B_{\ell}\right\}$ the collection of minimal authorized sets in $\mathcal{A}$. Let $\mathcal{C}=\left\{\left(C_{1,0}, C_{1,1}\right),\left(C_{2,0}, C_{2,1}\right)\right.$, $\left.\ldots,\left(C_{t, 0}, C_{t, 1}\right)\right\}$ be a collection of pairs of sets of parties. We say that $\mathcal{C}$ satisfies the unique intersection property for $\mathcal{A}$ if

1. For every $1 \leq j \leq t,\left\{p_{1}, \ldots, p_{n}\right\} \backslash\left(C_{j, 0} \cup C_{j, 1}\right) \notin \mathcal{A}$.

2. For every $1 \leq i \leq \ell$ and every $1 \leq j \leq t$, exactly one of the following conditions hold (1) $B_{i} \cap C_{j, 0} \neq \emptyset$, (2) $B_{i} \cap C_{j, 1} \neq \emptyset$.

Note that if $B \in \mathcal{A}$ and $\left\{p_{1}, \ldots, p_{n}\right\} \backslash C \notin \mathcal{A}$, then $B \cap C \neq \emptyset$ (otherwise, $B \subseteq\left\{p_{1}, \ldots, p_{n}\right\} \backslash C$, contradicting the monotonicity of $\mathcal{A}$ ). Thus, Condition (2) in Definition 4.4 requires that $B_{i}$ intersects at most one of the sets $C_{j, 0}, C_{j, 1}$.

Theorem 4.5. Let $\mathcal{C}$ be a collection satisfying the unique intersection property for $\mathcal{A}$. Define a matrix $D$ of size $\ell \times t$, with $D_{i, j}=0$ if $B_{i} \cap C_{i, 0} \neq \emptyset$ and $D_{i, j}=1$ if $B_{i} \cap C_{i, 1} \neq \emptyset$. Then, the size of every multi-target monotone span program accepting $\mathcal{A}$ is at least $\operatorname{rank}_{\mathbb{F}}(D)$.

Proof. Let $\mathcal{M}=\left(\mathbb{F}, M, \rho, X=\left\{\boldsymbol{e}_{1}, \ldots, \boldsymbol{e}_{k}\right\}\right)$ be a multi-target monotone span program accepting $\mathcal{A}$, and denote the number of rows of $M$ by $m$. For every $1 \leq i \leq \ell$ since $B_{i} \in \mathcal{A}$ the rows of $M$ labeled by the parties of $B_{i}$ span $X$. By Observation 4.2 for every $1 \leq r \leq k$, there exists $\boldsymbol{v}_{i, r}$ such that $\boldsymbol{v}_{i, r} M=\boldsymbol{e}_{r}$ and the non-zero coordinates of $\boldsymbol{v}_{i, r}$ are only in rows labeled by $B_{i}$.

Fix $1 \leq j \leq t$ and let $T_{j}=\left\{p_{1}, \ldots, p_{n}\right\} \backslash\left(C_{j, 0} \cup C_{j, 1}\right)$. Since $T_{j} \notin \mathcal{A}$, by Observation 4.3. for every $1 \leq s \leq k$ there exists a vector $\boldsymbol{w}_{j, s}$ such that $M_{T_{j}} \boldsymbol{w}_{j, s}=0$, $\boldsymbol{e}_{s} \cdot \boldsymbol{w}_{j, s}=1$ and $\forall r \neq s, \boldsymbol{e}_{\boldsymbol{r}} \cdot \boldsymbol{w}_{\boldsymbol{j}, \boldsymbol{s}}=0$. Let $\boldsymbol{y}_{j, s}:=M \boldsymbol{w}_{j, s}$ and define $\boldsymbol{z}_{j, s}$ to be the column vector achieved from $\boldsymbol{y}_{j, s}$ by replacing all coordinates in $\boldsymbol{y}_{j, s}$ labeled by parties in $C_{j, 0}$ with zero. The only non-zero coordinates in $\boldsymbol{z}_{j, s}$ are in coordinates labeled by $C_{j, 1}$.

Define $L$ as the matrix with rows $v_{1,1}, \ldots, v_{\ell, 1}, v_{1,2}, \ldots, v_{\ell, 2}, \ldots, v_{\ell, k}$ and $R$ the matrix with columns $z_{1,1}, \ldots, z_{\ell, 1}, z_{1,2}, \ldots, z_{\ell, 2}, \ldots, z_{\ell, k}$. Note that by definition the rows of $L$ are of length $m$, so $L$ has $m$ columns, thus, $\operatorname{rank}(L) \leq m$.

Let $\mathbf{D}=L R$. We next prove that $\mathbf{D}$ is a block matrix of the form:

$$
\mathbf{D}=\left[\begin{array}{cccc}
D & 0 & \ldots & 0 \\
0 & D & \ldots & 0 \\
\vdots & \vdots & \ddots & \vdots \\
0 & 0 & \ldots & D
\end{array}\right]
$$

where $D$ is the matrix defined in the Theorem. We need to show that $\boldsymbol{v}_{i, r} \cdot \boldsymbol{z}_{s, j}=0$ if $r \neq s$ (off the diagonal matrix block) and $\boldsymbol{v}_{i, r} \cdot \boldsymbol{z}_{s, j}=D_{i, j}$ if $r=s$.

- If $B_{i} \cap C_{j, 0} \neq \emptyset, D_{i, j}=0$. Furthermore, $B_{i} \cap C_{j}, 1=\emptyset$, thus, $\boldsymbol{v}_{i, r}$ and $\boldsymbol{z}_{s, j}$ do not share non-zero coordinates and $\boldsymbol{v}_{i, r} \cdot \boldsymbol{z}_{s, j}=0$. In particular, if $r=s$ then $\boldsymbol{v}_{i, r} \cdot \boldsymbol{z}_{r, j}=0=D_{i, j}$, and if $r \neq s$ then $\boldsymbol{v}_{i, r} \cdot \boldsymbol{z}_{s, j}=0$ as desired.

- If $B_{i} \cap C_{j, 1} \neq \emptyset$, then $D_{i, j}=1, B_{i} \cap C_{j, 0}=\emptyset$, and all coordinates in $\boldsymbol{v}_{i, r}$ labeled by $C_{j, 0}$ are zero, thus,

$$
\boldsymbol{v}_{i, r} \cdot \boldsymbol{z}_{s, j}=\boldsymbol{v}_{i, r} \cdot \boldsymbol{y}_{s, j}=\boldsymbol{v}_{i, r} M \boldsymbol{w}_{s, j}=\boldsymbol{e}_{r} \cdot \boldsymbol{w}_{s, j}=\left\{\begin{array}{l}
0 r \neq s \\
1 r=s
\end{array}\right.
$$


In particular, if $r=s$ then $\boldsymbol{v}_{i, r} \cdot \boldsymbol{z}_{r, j}=1=D_{i, j}$ and if $r \neq s$ then $\boldsymbol{v}_{i, r} \cdot \boldsymbol{z}_{s, j}=0$. So $\operatorname{rank}_{\mathbb{F}}(\mathbf{D})=k \cdot \operatorname{rank}_{\mathbb{F}}(D)$, and since $\mathcal{M}$ is a $k$-linear representation, its size is $\frac{m}{k} \geq \frac{\operatorname{rank}_{\mathbb{F}}(L)}{k} \geq \frac{\operatorname{rank}_{\mathbb{F}}(\mathbf{D})}{k}=\operatorname{rank}_{\mathbb{F}}(D)$.

By [17, for every $n$ there is an access structure $\mathcal{A}$ with $n$ parties, for which there exists a collection $\mathcal{C}$ satisfying the unique intersection property, such that $\operatorname{rank}_{\mathbb{F}}(D) \geq n^{\Omega(\log n)}$ (where $D$ is as defined in Theorem 4.5). So by Theorem 4.5 .

Corollary 4.6. For every $n$, there exists an access structure $\mathcal{N}_{n}$ with $n$ parties such that every multi-target monotone span program over any field accepting it has size $n^{\Omega(\log n)}$.

As multi-target monotone span program are equivalent to multi-linear secretsharing schemes [20], the same lower bound applies to multi-linear secret-sharing schemes.

Corollary 4.7. For every $n$, there exists an access structure $\mathcal{N}_{n}$ with $n$ parties such that the information ratio of every multi-linear secret-sharing scheme realizing it is $n^{\Omega(\log n)}$.

\section{References}

1. Babai, L., Gál, A., Wigderson, A.: Superpolynomial lower bounds for monotone span programs. Combinatorica 19(3), 301-319 (1999)

2. Ben-Or, M., Goldwasser, S., Wigderson, A.: Completeness theorems for noncryptographic fault-tolerant distributed computations. In: Proc. of the 20th ACM Symp. on the Theory of Computing, pp. 1-10 (1988)

3. Benaloh, J., Leichter, J.: Generalized secret sharing and monotone functions. In: Goldwasser, S. (ed.) CRYPTO 1988. LNCS, vol. 403, pp. 27-35. Springer, Heidelberg (1990)

4. Bertilsson, M., Ingemarsson, I.: A construction of practical secret sharing schemes using linear block codes. In: Zheng, Y., Seberry, J. (eds.) AUSCRYPT 1992. LNCS, vol. 718, pp. 67-79. Springer, Heidelberg (1993)

5. Blakley, G.R.: Safeguarding cryptographic keys. In: Merwin, R.E., Zanca, J.T., Smith, M. (eds.) Proc. of the 1979 AFIPS National Computer Conference. AFIPS Conference proceedings, vol. 48, pp. 313-317. AFIPS Press (1979)

6. Blundo, C., De Santis, A., Stinson, D.R., Vaccaro, U.: Graph decompositions and secret sharing schemes. J. Cryptology 8(1), 39-64 (1995)

7. Brickell, E.F.: Some ideal secret sharing schemes. Journal of Combin. Math. and Combin. Comput. 6, 105-113 (1989)

8. Brickell, E.F., Davenport, D.M.: On the classification of ideal secret sharing schemes. J. of Cryptology 4(73), 123-134 (1991)

9. Chaum, D., Crépeau, C., Damgård, I.: Multiparty unconditionally secure protocols. In: Proc. of the 20th ACM Symp. on the Theory of Computing, pp. 11-19 (1988) 
10. Cramer, R., Damgård, I., Maurer, U.: General secure multi-party computation from any linear secret-sharing scheme. In: Preneel, B. (ed.) EUROCRYPT 2000. LNCS, vol. 1807, pp. 316-334. Springer, Heidelberg (2000)

11. Csirmaz, L.: The size of a share must be large. J. of Cryptology 10(4), 223-231 (1997)

12. Desmedt, Y., Frankel, Y.: Shared generation of authenticators and signatures. In: Feigenbaum, J. (ed.) CRYPTO 1991. LNCS, vol. 576, pp. 457-469. Springer, Heidelberg (1992)

13. van Dijk, M.: A linear construction of secret sharing schemes. Designs, Codes and Cryptography 12(2), 161-201 (1997)

14. Dowling, T.A.: A class of geometric lattices based on finite groups. J. Comb. Theory, Ser. B 14(1), 61-86 (1973)

15. Dowling, T.A.: A q-analog of the partition lattice. A Survey of Combinatorial Theory, 101-115 (1973)

16. Gál, A.: A characterization of span program size and improved lower bounds for monotone span programs. Computational Complexity 10(4), 277-296 (2001)

17. Gál, A., Pudlák, P.: A note on monotone complexity and the rank of matrices. Inform. Process. Lett. 87, 321-326 (2003)

18. Goyal, V., Pandey, O., Sahai, A., Waters, B.: Attribute-based encryption for finegrained access control of encrypted data. In: Proc. of the 13th ACM Conference on Computer and Communications Security, pp. 89-98 (2006)

19. Ito, M., Saito, A., Nishizeki, T.: Secret sharing schemes realizing general access structure. In: Proc. of the IEEE Global Telecommunication Conf., Globecom 1987, pp. 99-102 (1987); Journal version: Multiple assignment scheme for sharing secret. J. of Cryptology 6(1), 15-20 (1993)

20. Karchmer, M., Wigderson, A.: On span programs. In: Proc. of the 8th IEEE Structure in Complexity Theory, pp. 102-111 (1993)

21. Karnin, E.D., Greene, J.W., Hellman, M.E.: On secret sharing systems. IEEE Trans. on Information Theory 29(1), 35-41 (1983)

22. Linnik, Y.V.: On the least prime in an arithmetic progression I. the basic theorem. Rec. Math (Mat. Sbornik) N.S. 15(57), 139-178 (1944)

23. Linnik, Y.V.: On the least prime in an arithmetic progression II. the deuringheilbronn phenomenon. Rec. Math (Mat. Sbornik) N.S. 15(57), 347-368 (1944)

24. Martí-Farré, J., Padró, C.: On secret sharing schemes, matroids and polymatroids. Journal of Mathematical Cryptology 4(2), 95-120 (2010)

25. Matúš, F.: Matroid representations by partitions. Discrete Mathematics 203, 169-194 (1999)

26. Milne, J.S.: Group theory, v3.12 (2012), http://www.jmilne.org/math/

27. Naor, M., Wool, A.: Access control and signatures via quorum secret sharing. In: 3rd ACM Conf. on Computer and Communications Security, pp. 157-167 (1996)

28. Oxley, J.G.: Matroid Theory, 2nd edn. Oxford University Press (2011)

29. Pendavingh, R.A., van Zwam, S.H.M.: Skew partial fields, multilinear representations of matroids, and a matrix tree theorem. Advances in Applied Mathematics 50(1), 201-227 (2013)

30. Semple, C., Whittle, G.: Partial fields and matroid representation. Advances in Applied Mathematics 17(2), 184-208 (1996)

31. Serre, J.-P.: Linear Representations of Finite Groups. Springer (1977)

32. Seymour, P.D.: On secret-sharing matroids. J. of Combinatorial Theory, Series B 56, 69-73 (1992) 
33. Shamir, A.: How to share a secret. Communications of the ACM 22, 612-613 (1979)

34. Shankar, B., Srinathan, K., Rangan, C.P.: Alternative protocols for generalized oblivious transfer. In: Rao, S., Chatterjee, M., Jayanti, P., Murthy, C.S.R., Saha, S.K. (eds.) ICDCN 2008. LNCS, vol. 4904, pp. 304-309. Springer, Heidelberg (2008)

35. Simonis, J., Ashikhmin, A.: Almost affine codes. Designs, Codes and Cryptography 14(2), 179-197 (1998)

36. Stinson, D.R.: Decomposition construction for secret sharing schemes. IEEE Trans. on Information Theory 40(1), 118-125 (1994)

37. Tassa, T.: Generalized oblivious transfer by secret sharing. Des. Codes Cryptography 58(1), 11-21 (2011)

38. van Dijk, M., Jackson, W.-A., Martin, K.M.: A general decomposition construction for incomplete secret sharing schemes. Des. Codes Cryptography 15(3), 301-321 (1998)

39. van Dijk, M., Kevenaar, T.A.M., Schrijen, G.J., Tuyls, P.: Improved constructions of secret sharing schemes by applying (lambda, omega)-decompositions. Inform. Process. Lett. 99(4), 154-157 (2006)

40. Vincent, G.: Les groupes lineaires finis sans point fixes. Commentarii Mathematici Helvetici 20, 117-171 (1947)

41. Waters, B.: Ciphertext-policy attribute-based encryption: An expressive, efficient, and provably secure realization. In: Catalano, D., Fazio, N., Gennaro, R., Nicolosi, A. (eds.) PKC 2011. LNCS, vol. 6571, pp. 53-70. Springer, Heidelberg (2011)

42. Wolf, J.A.: Spaces of Constant Curvature, 5th edn. Publish or Perish, Inc. (1984)

43. Xylouris, T.: On the least prime in an arithmetic progression and estimates for the zeros of Dirichlet $L$-functions. Acta Arith. 150(1), 65-91 (2011)

44. Zassenhaus, H.: Uber endliche faskorper. Abhandlungen aus dem Mathematischen Seminar der Hamburgischen Universitat 11, 187-220 (1935)

\section{A The Construction of the Group $G_{p}$}

In this section we briefly explain the construction of the group $G_{p}$ (for any prime $p$ ), which appears in Proposition 2.15. We then give a partial explanation of why any fixed-point free representation of $G_{p}$ is of dimension at least $p$. The complete proofs, and more details on the construction, will be given in the full version.

Semidirect products. We assume some familiarity with group basics, such as group homomorphisms and automorphisms. Let $N$ be a group. Recall that the set of all automorphisms of $N$, denoted $\operatorname{Aut}(N)$, is also a group, with group operation being composition, and the identity element being the identity map. We now recall the definition of an action of a group $H$ on a group $N$.

Definition A.1. Let $H$ and $N$ be two groups. By an action of $H$ on $N$, denoted $H \curvearrowright N$, we mean a group homomorphism $\phi: H \rightarrow \operatorname{Aut}(N)$.

To simplify the notation, if no confusion is possible, we use shorter notation $x^{g}:=(\phi(g))(x)$. Since $\phi$ is a homomorphism, the identity of $G$ is mapped to the identity automorphism.

Example A.2. For any pair of groups $H$ and $N$, there always exists the trivial action $\tau: H \rightarrow \operatorname{Aut}(N)$, which maps every element of $H$ to the identity automorphism. A non-trivial action, however, does not always exist, and depends on the choice of $H$ and $N$. 
Example A.3. Let $H=\mathbb{Z}_{2}$ and $N=\mathbb{Z}_{3}$. To avoid confusion we denote $N=$ $\{0,1,2\}$ and $H=\left\{f_{0}, f_{1}\right\}$. Then $H$ acts on $N$ by $\phi\left(f_{1}\right)(1)=2$. We note that this completely identifies the action because $f_{1}$ and 1 are generators of $H$ and $N$ respectively. Thus, for example, $f_{1}(2)=f_{1}(1+1)=f_{1}(1)+f_{1}(1)=2+2=1$ and $f_{0}(1)=f_{1} \circ f_{1}(1)=f_{1}(2)=1$. So it remains to verify only that this is well defined, which is a very small task.

Lemma A.4. Let $\psi: G_{1} \rightarrow G_{2}$ be a group homomorphism, and $\phi: G_{2} \curvearrowright N$ a group action. Then $\psi$ induces a group action $\psi^{*}(\phi): G_{1} \curvearrowright N$, given by composition $\left(\psi^{*}(\phi)\right)(x):=\phi(\psi(x))$. Furthermore, if $\psi$ is surjective and the action $\phi$ is non-trivial then so is $\psi^{*}(\phi)$.

Proof. Follows easily from the definitions.

The following proposition is well known.

Proposition A.5. For any prime $q$, the group of automorphisms of $\mathbb{Z}_{q}$ is isomorphic to the group $\mathbb{Z}_{q-1}$.

This allows us to build a non-trivial action of $\mathbb{Z}_{p}$ on $\mathbb{Z}_{q}$, if $p, q$ are primes such that $q \equiv 1 \bmod p$.

Proposition A.6. Let $p, q \in \mathbb{N}$ be two primes such that $q \equiv 1 \bmod p$. Then $\mathbb{Z}_{p}$ admits a non-trivial action on $\mathbb{Z}_{q}$.

Proof. From Proposition A.5 $A u t\left(\mathbb{Z}_{q}\right) \simeq \mathbb{Z}_{q-1}$. Thus, it suffices to construct a non-trivial homomorphism $\phi: \mathbb{Z}_{p} \rightarrow \mathbb{Z}_{q-1}$. Let $n \in \mathbb{N}$ be such that $q-1=n p$, and set $\phi(x):=n x \bmod q$. Then $\phi$ is a non-trivial homomorphism.

Corollary A.7. Let $p, q$ be as in Proposition A.6. Then $\mathbb{Z}_{p^{2}}$ admits a non-trivial action on $\mathbb{Z}_{q}$.

Proof. We have a natural surjective homomorphism $\psi: \mathbb{Z}_{p^{2}} \rightarrow \mathbb{Z}_{p}$ given by $\psi(x):=x \bmod p$. Thus, by Proposition A.6 and Lemma A.4, $\psi^{*}(\phi)$ is a nontrivial action of $\mathbb{Z}_{p^{2}}$ on $\mathbb{Z}_{q}$.

There may exist other non-trivial actions of $\mathbb{Z}_{p^{2}}$ on $\mathbb{Z}_{q}$. However, from now on when we mention the action of $\mathbb{Z}_{p^{2}}$ on $\mathbb{Z}_{q}$, we mean that we have fixed an isomorphism $\operatorname{Aut}\left(\mathbb{Z}_{q}\right) \simeq \mathbb{Z}_{q-1}$ and we refer to the non-trivial action $\psi^{*}(\phi)$ constructed in the proof of Corollary A.7.

Definition A.8. Let $H$ be a group acting on another group $N$, and $\phi: H \rightarrow$ Aut $(N)$ the action. The semidirect product, denoted $N \rtimes_{\phi} H$, is the set $N \times H=$ $\{(n, h) \mid n \in N, h \in H\}$ equipped with the following operation

$$
\left(n_{1}, h_{1}\right) \cdot\left(n_{2}, h_{2}\right):=\left(n_{1} \cdot n_{2}^{h_{1}}, h_{1} \cdot h_{2}\right) .
$$

We leave to the reader to verify that (13) indeed defines a group-law. We will often omit $\phi$ in the notation of the semidirect product, and write simply $N \rtimes G$. When the action of $G$ on $N$ is not trivial we will say that the semidirect product is non-trivial. An attractive property of non-trivial semidirect products is that they are not abelian, even if $H$ and $N$ are. 
Lemma A.9. If $N \rtimes G$ is a non-trivial semidirect product then it is not abelian.

Proof. Since $G$ acts non-trivially, there exist $g \in G$ and $h \in N$ such that $h^{g} \neq h$. Therefore $\left(e_{N}, g\right) \cdot\left(h, e_{G}\right)=\left(h^{g}, g\right) \neq(h, g)=\left(h, e_{G}\right) \cdot\left(e_{N}, g\right)$.

Proposition A.10. Let $p$ and $q$ be prime integers satisfying $q \equiv 1 \bmod p$. Then there exists a non-trivial semidirect product $G_{p}=\mathbb{Z}_{q} \rtimes \mathbb{Z}_{p^{2}}$. The group has $p^{2} \cdot q$ elements.

Proof. Follows immediately from the definitions and Corollary A.7.

Suitability of $G_{p}$. We now explain why the above construction works for us. Since a full proof requires quite a few pages of background in representation theory, we will only give a brief overview and refrain from proving the following claims, which rely on some classical theorems in representation theory. But first we state Linnik's theorem:

Theorem A.11 (Linnik's Theorem). There exists constants $c, L$ such that for any pair of co-prime integers $a$ and $d$, with $1 \leq a<d$, the smallest prime of the form $a+n d(n \geq 1)$ is smaller than $c d^{L}$.

Linnik didn't give an explicit bound on $L$, but later works have shown that $L$ is in fact very small. The current state of the art is $L \leq 5.18$ due to Xylouris [43].

Corollary A.12. For every prime $p$, there exists a prime $q$, with $q=O\left(p^{5.18}\right)$, for which a non-trivial semidirect product $\mathbb{Z}_{q} \rtimes \mathbb{Z}_{p^{2}}$ exists.

Now fix a prime $p$ and a prime $q$ such that $q \equiv 1 \bmod p$, and let $G_{p}=\mathbb{Z}_{q} \rtimes \mathbb{Z}_{p^{2}}$ be the non-trivial semidirect product explained above.

Lemma A.13. The group $G_{p}$ is solvable and every proper subgroup of $G_{p}$ is cyclic. Thus, from the classification of solvable fixed-point free groups (see for example [42, Theorem 6.1.11]), the group $G_{p}$ admits a fixed-point free representation.

Lemma A.14. The group $G_{p}$ is not abelian. This implies that $G_{p}$ does not have fixed-point free representations of dimension 1.

Lemma A.15. The group $G_{p}$ does not have any fixed-point free representations over fields of characteristic $p, q$.

Lemma A.16. Over fields of characteristic different from $p, q$, the dimension of the smallest fixed-point representation of $G_{p}$ divides the order of $G_{p}$. Thus, since the dimension cannot be $1, G_{p}$ has a fixed-point free representation of dimension $\geq p$.

The completion of Proposition 2.15 (i.e., bounding the size of the field) is done by explicitly building a fixed-point free representation of $G_{p}$ of dimension $p$ over the field $\mathbb{F}_{2^{p q}}$. 